\title{
New Microscopy - the Imaging of Quantum Materials
}

\author{
David C. Bell, Estelle Kalfon-Cohen and Robert M. Westervelt
}

School of Engineering and Applied Sciences, Harvard University, Cambridge, MA 02138

The discovery of extraordinary new quantum materials with striking properties has caused great excitement, and promises to transform signal processing and computation. We have performed integrated research on three materials (1) Graphene $(\mathrm{G})$ - electrons that move as massless particles at a constant speed; (2) Topological Insulators (TI) - mobile surface electrons with spins fixed to the direction of motion; and (3) Nitrogen-vacancy (NV) Centers in Diamond - a single spin stores a bit of quantum information. Remarkably, the quantum phenomena displayed by these materials persists at room temperature, changing the rules for signal processing and computation and opening the way for quantum electronics. Electrons and holes in graphene and topological insulators act in ways that are totally unlike carriers in conventional semiconductors -they move like two-dimensional (2D) massless, ultra-relativistic electrons, except their speed is much less than the speed of light.

These quantum materials are ideally suited to layered atomic-scale structures that control the flow of charge and spin in graphene and TI materials, with memory and optical input/output channels provided by NV-centers in diamond. We have imaged and characterized high quality graphene and graphene-like materials, such as hexagonal boron nitride $(\mathrm{hBN})$ and hybrid graphene-hBN structures. Until now, most experimental demonstrations of the remarkable properties of graphene have been done using exfoliated graphene sheets. Compared with mechanical exfoliation, CVD synthesis [1-2] can provide larger areas, with wafer-scale monolayer or multilayer graphene sheets. Moreover, the atomic configuration along the edge of a graphene sheet can have a large effect on its electronic properties and create new electronic states [3]. A major thrust of this research has been to discover new ways to image these materials and characterize graphene and $\mathrm{hBN}$ sheets using TEM imaging (Fig.1). We have imaged $\mathrm{Bi}_{2} \mathrm{Te}_{2} \mathrm{Se}$, which has a lower bulk carrier density and higher mobility than $\mathrm{Bi}_{2} \mathrm{Se}_{3}$. Aberration-corrected electron microscopy has been used to characterize MBE-grown films with high resolution at low beam voltages $(40 \& 80 \mathrm{kV})$ to directly visualize structural defects and relate them to performance.

The imaging and analysis of quantum materials presents new challenges on how to minimize surface and sample damage while imaging and analyzing structures at the direct atomic level the ability to image the interface (Fig. 2) between two types of quantum materials is critical to understand the coupling mechanisms for information transfer between them

\section{References:}

[1] A. Reina, X. Jia, J. Ho, D. Nezich, H. Son, V. Bulovic, M.S. Dresselhaus, \& J. Kong, Nano Lett. 9, 30-35 (2008).

[2] K.S. Kim, Y. Zhao, H. Jang, S.Y. Lee, J.M. Kim, K.S. Kim, J.H. Ahn, P. Kim, J.Y. Choi, and B.H. Hong, Nature 457, 706-710 (2009).

[3] K Nakada, M Fujita, G. Dresselhaus and M. S Dresselhaus, Phy. Rev. B., 54 (1996), 17954.

[4] This work was supported by the STC Center for Integrated Quantum Materials, NSF Grant No. DMR-1231319. 


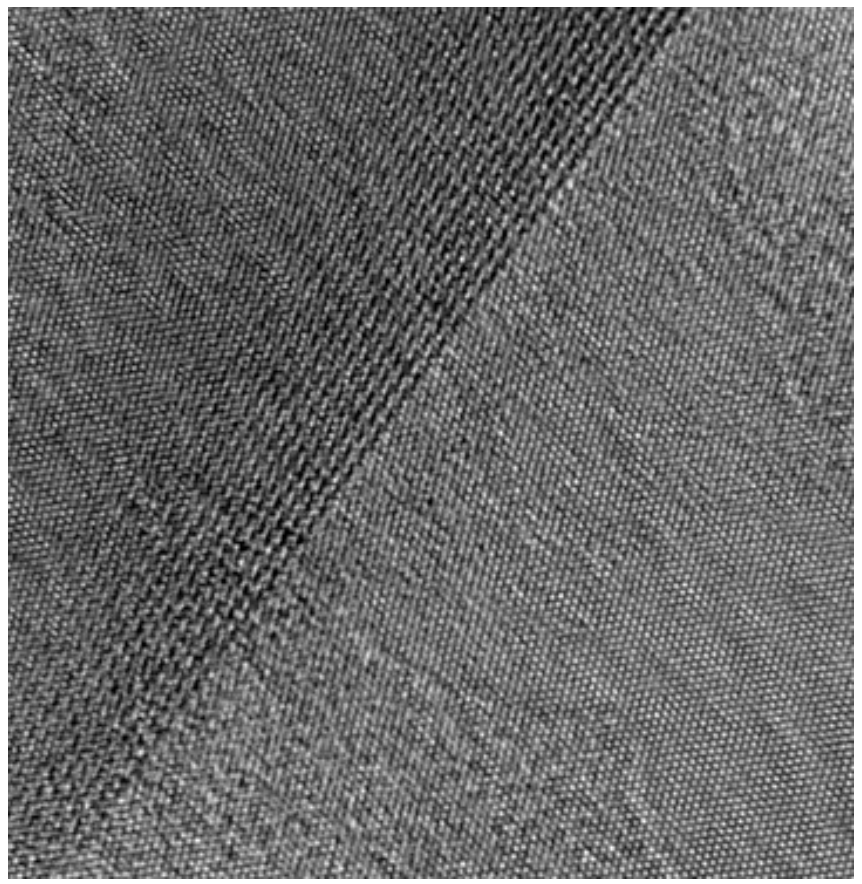

Figure 1. $80 \mathrm{keV}$ Aberration corrected TEM image of a multilayered graphene structure.

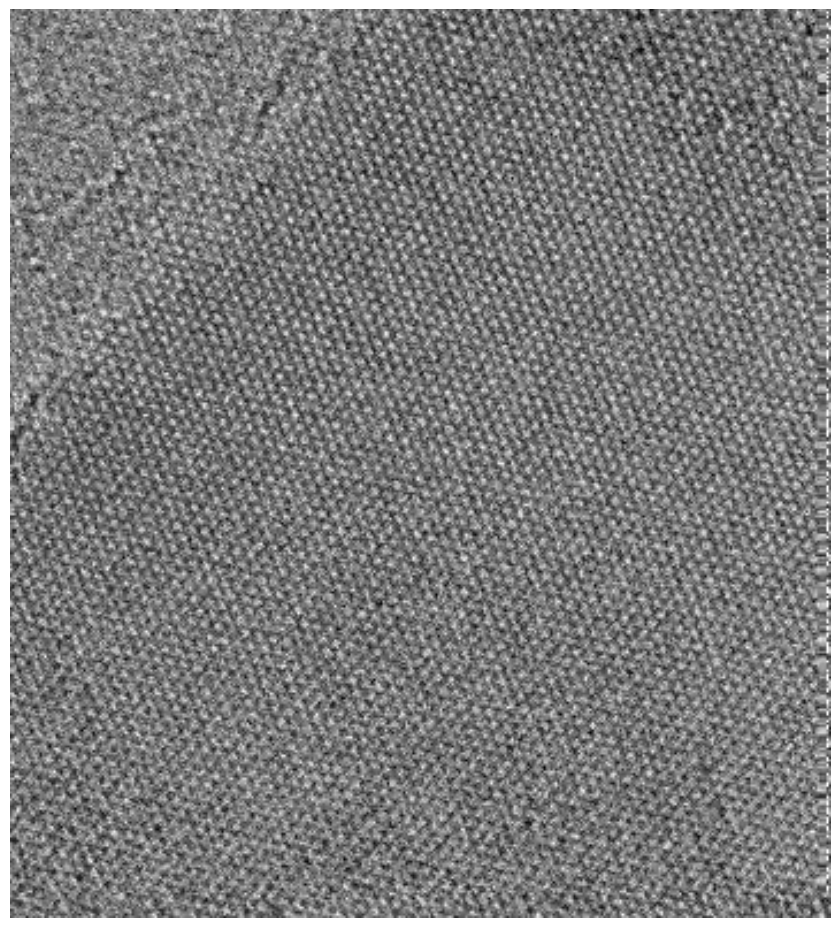

Figure 2. Example, high-resolution image of a graphene \& BN interface. 